\begin{tabular}{|c|l|}
\hline Title & $\begin{array}{l}\text { Transition from convection rolls to large scale cellular structures in turbulent Rayleigh-Benard convection in a liquid } \\
\text { metal lay er }\end{array}$ \\
\hline Author(s) & A kashi, Megumi; Y anagisawa, Takatoshi; Tasaka, Y uji; V ogt, Tobias; Murai, Y Yichi; Eckert, Sven \\
\hline Citation & $\begin{array}{l}\text { Physical Review Fluids, 4(3), 033501 } \\
\text { https://doi.org/40.1103PhysRevFluids.4.033501 }\end{array}$ \\
\hline Issue Date & 2019-03-08 \\
\hline Doc URL & http://hdl.handle.net/2115/73528 \\
\hline Rights & @2019A A merican Physical Society \\
\hline Type & article \\
\hline File Information & PhysRevFluids.4.033501.pdf \\
\hline
\end{tabular}

Instructions for use 


\title{
Transition from convection rolls to large-scale cellular structures in turbulent Rayleigh-Bénard convection in a liquid metal layer
}

\author{
Megumi Akashi, ${ }^{1,2}$ Takatoshi Yanagisawa, ${ }^{3,1}$ Yuji Tasaka, ${ }^{1,}$ Tobias Vogt, ${ }^{2}$ \\ Yuichi Murai, ${ }^{1}$ and Sven Eckert ${ }^{2}$ \\ ${ }^{1}$ Laboratory for Flow Control, Hokkaido University, Sapporo 060-8638, Japan \\ ${ }^{2}$ Helmholtz Zentrum Dresden-Rossendorf (HZDR), Dresden 01328, Germany \\ ${ }^{3}$ Japan Agency for Marine-Earth Science and Technology (JAMSTEC), Yokosuka 237-0061, Japan
}

(Received 3 August 2018; published 8 March 2019)

\begin{abstract}
Turbulent Rayleigh-Bénard convection was investigated within a liquid metal layer, Prandtl number $\operatorname{Pr}=0.03$, in a square vessel having a moderate aspect ratio, $\Gamma=5$. Laboratory experiments were performed at moderate Rayleigh numbers, $7.9 \times 10^{3}<$ $\mathrm{Ra}<3.5 \times 10^{5}$. Ultrasonic velocity profiling was used to visualize the spatiotemporal flow structure in two horizontal planes, while temperature fluctuations were monitored simultaneously in the fluid layer. By using multiple ultrasonic sensors, a grid of orthogonal measurement lines was created. This configuration enabled the identification of coherent flow structures showing periodic oscillations. In particular, oscillatory roll-like structures were observed for $\mathrm{Ra} \lesssim 6 \times 10^{4}$, while the transition to a new-found, fully three-dimensional cellular structure occurs around $\mathrm{Ra}=7 \times 10^{4}$. The Fourier analysis of the temperature fluctuations indicates that the convection reaches the developed state of thermal turbulence at this Ra number. This cellular structure of the flow field is recognized as a representation of the large-scale circulation in thermal turbulence for the specific situation of confined convection in the rectangular vessel. The transition from laminar convection to thermal turbulence manifests itself in the occurrence of unstable intermediate regimes accompanied by a stepwise increment in the horizontal scale. We suggest scaling laws for the characteristic velocity and the dominating oscillation frequency and based on that for the horizontal length scale as a function of the Ra number. The comparison to corresponding values of characteristic length scales published for thermal convection in air in larger aspect ratios [Pr $=0.7$, T. Hartlep et al., Phys. Rev. Lett. 91, 064501 (2003), A. Pandey et al., Nat. Commun. 9, 2118 (2018), and D. E. Fitzjarrald, J. Fluid Mech. 73, 693 (1976)] reveals a different Ra number dependence of the horizontal wave number.
\end{abstract}

DOI: 10.1103/PhysRevFluids.4.033501

\section{INTRODUCTION}

Rayleigh-Bénard convection (RBC) designates buoyancy-driven flows in a fluid layer heated from below and cooled from above. It is a classical problem in fluid dynamics and can be taken as a basic configuration for various issues in geophysics, astrophysics, and engineering. The RBC system is dominated by two dimensionless control parameters: the Rayleigh number, $\mathrm{Ra}=\alpha g \Delta T L^{3} /(\kappa \nu)$, and the Prandtl number, $\operatorname{Pr}=v / \kappa$, where $\alpha, \kappa$, and $v$ denote the thermal expansion coefficient, thermal diffusivity, and kinematic viscosity of test fluids, respectively. The symbols $g$ and $\Delta T$ stand for the gravitational acceleration and the vertical temperature difference, respectively, in the fluid layer with a thickness $L$. Different convective flow patterns can be found in the Ra-Pr parameter

*tasaka@eng.hokudai.ac.jp 
space. Thermal turbulence appears at sufficiently large Ra numbers, while it is widely expected that the flow tends to be more turbulent the smaller the Pr number (cf. Refs. [1,2]).

It is well known that under the condition of thermal turbulence a mean flow is spontaneously formed in a finite fluid layer. The existence of such flow structures, termed "large-scale circulation (LSC)," was first reported by Krishnamurti and Howard [2]. The LSC is driven by systematic drift of transient thermal plumes and is therefore not to be confused with the typical convection rolls that form directly after the onset of thermal convection. It is obvious that the structure and the properties of the LSC should depend on both the Ra and the Pr number as well as the geometry of the convection vessel. The spatiotemporal characteristics of the LSC have been investigated mainly for water $(\operatorname{Pr} \sim 7)$ and air $(\operatorname{Pr} \sim 0.7)$ layers $[3,4]$. Most of the previous studies are restricted to aspect ratios of the fluid layer $(\Gamma)$ around unity at which a single-roll LSC occupies the entire fluid layer. Recently, several examples of thermal turbulence examined in fluid layers with $\Gamma \gg 1$ have been reported. From numerical simulations it is reported that the typical size of the coherent flow structures in an air layer at $\Gamma=10$ increases discretely with increasing Ra [5]. Hartlep et al. [5] also mentioned that the large-scale structures are continuations of the flow pattern formed in laminar conditions at smaller Ra numbers. Von Hardenberg et al. [6] performed numerical simulations of convection in air with $2 \pi \leqslant \Gamma \leqslant 12 \pi$ and showed that there is a finite horizontal saturation scale of large-scale patterns for sufficiently large $\Gamma$ in the range $10^{5} \leqslant \mathrm{Ra} \leqslant 10^{8}$. By numerical simulation in a shallow cylindrical vessel with $\Gamma \sim 50$, Emran and Schumacher [7] demonstrated that time-averaged streamlines at $\mathrm{Ra}=5 \times 10^{5}$ reveal organized convection patterns very similar to those observed at a slightly supercritical $\mathrm{Ra}$ number of $5 \times 10^{3}$, while the instantaneous streamlines appear complex and disorganized. Bailon-Cuba et al. [8] investigated heat transport and corresponding transitions of flow patterns in thermal turbulence in an air layer as a function of $\Gamma(0.5 \leqslant \Gamma \leqslant 12)$; the LSC exhibits multiple roll structures or hexagonal cellular structures similar to those observed at slightly supercritical conditions close to the onset of convection for sufficiently large aspect ratios $(\Gamma \geqslant 8)$. For lager Pr numbers, $\operatorname{Pr}=6.3$, at $\operatorname{Ra}=9.6 \times 10^{7}$, numerical simulations performed by Sakievich et al. [9] showed the formation of hub-and-spoke structures as long-living coherent structures in a cylindrical domain at $\Gamma=6.3$. Pandey et al. [10] investigated large-scale structures on lengths larger than the height of the fluid layer at different $\mathrm{Pr}$ numbers ranging from 0.005 to 70 and Ra numbers up to $10^{7}$ in a square cell of aspect ratio 25 . Their analysis demonstrates a distinct dependence of the characteristic length scales of the large-scale order structure on both the Pr and the Ra numbers and suggests the existence of a description of turbulent superstructures in thermally driven convection. However, the Ra number dependence of the typical length scales was not shown for small $\operatorname{Pr}$ numbers $(\operatorname{Pr}<0.7)$ yet.

Krishnamurti [1] investigated the transition from steady to time-dependent flow at a slightly supercritical $\mathrm{Ra}$ number by temperature fluctuations in a fluid layer of mercury $(\operatorname{Pr}=0.024)$. Further increase of Ra leads to the transition to turbulence, while in the case of small Pr numbers the transition occurs at lower Ra numbers [1,2]. The so-called "Busse balloon" describes the stable region in the Ra-wave-number plane where two-dimensional (2D) steady roll structures occur after the onset of convection. This domain of stability, together with the style of instabilities bounding the domain, strongly depends on the Pr number [11]. Laboratory experiments $[1,12]$ came to the conclusion that the Busse balloon shrinks to a very narrow domain for very low-Pr numbers, $\operatorname{Pr} \sim O\left(10^{-2}\right)$. Exceeding the region of the Busse balloon by increasing the Ra number at these Pr numbers, theoretical studies showed that the pattern of convection rolls becomes time dependent due to the oscillatory instability at slightly supercritical $\mathrm{Ra}$, and traveling waves propagate in the direction of the roll axis [13-15]. The effect of traveling waves on the roll structure beyond the boundary of the Busse balloon was studied by numerical simulations [16,17].

The evolution of the LSC structure in low-Pr convection on the way to thermal turbulence is not fully understood yet. This situation provides the motivation for the experimental study on convection in a liquid metal layer presented here. Due to the limited capabilities to measure fluid velocities in liquid metals most of the previous experimental studies on liquid metal convection have limited themselves to the heat transfer in the system and the measurements of temperatures and their 
fluctuations [18-23]. Simultaneous measurement of velocity profiles and temperature fluctuations could provide a better understanding of the development and properties of LSCs in low-Pr thermal turbulence.

The ultrasonic velocity profiling (UVP) $[24,25]$ is the most straightforward tool to measure and visualize flow fields of opaque fluids such as liquid metals. It has already been applied for investigations of low-Pr thermal turbulence. The first measurements of instantaneous velocity distributions in liquid metal RBC were reported by Mashiko et al. [26] for a cylinder $(\Gamma=1)$ filled with mercury. Tsuji et al. [27] detected various complex modes of LSCs undergoing periodic oscillations in cylindrical geometries of aspect ratios around unity $(\Gamma=0.5,1$ and 2$)$ in the range of large Ra numbers $\left(10^{6}<\mathrm{Ra}<10^{10}\right)$. Yanagisawa et al. [28] studied flow patterns occurring in liquid gallium in a rectangular vessel $(200 \times 50 \times 40 \mathrm{~mm})$ and showed the existence of oscillatory roll structures up to $\mathrm{Ra} \sim 10^{5}$. In this configuration, the direction of rolls was strictly constrained by the geometry of the vessel, resulting in an orientation of the roll axes parallel to the shorter horizontal length of the vessel.

The main objective of this study is to identify and characterize coherent flow structures in low-Pr turbulent convection inside a square box $(\Gamma=5)$ at moderate Ra numbers, $O\left(10^{4}\right)-O\left(10^{5}\right)$. The focus is particularly on the evolution of the roll-like flow regimes, their structures are closely related to the commonly known convection rolls at the onset of convection, with increasing Ra number. For the highest Ra numbers in this study, it can be expected that the flow properties will comply with the state of thermal turbulence. In addition, it can be assumed that at sufficiently high Ra numbers a transition to cellular structures takes place, whose features resemble essential properties of turbulent superstructures. For the first time, we intend to establish a Ra number scaling for the characteristic wave length of the coherent structures in liquid metals at low-Pr numbers.

Multiple orthogonally superimposed horizontal velocity profiles are measured in the fluid layer at different heights by UVP. The measured data allow for identifying the large-scale three-dimensional (3D) flow structures. Thermocouples are used to monitor the corresponding temperature fluctuations simultaneously. Following the details of experimental setup and procedure explained in Sec. II, the development of spatiotemporal characteristics of the LSC with increasing Ra is investigated in Secs. III A, III B, and III C. The statistical turbulent properties of the convection are evaluated on the basis of the measured temperature fluctuations in Sec. III D. In Sec. IV A a scaling law describing the variation of the size of typical flow structures will be suggested and compared to the results obtained in different aspect ratios and Pr numbers $(0.005<\operatorname{Pr}<0.7)[5,10]$ and experiments performed in air layers at high aspect ratios [29]. Variations of horizontal scale of the coherent structures are further discussed in Sec. IV B.

\section{EXPERIMENTAL SETUP}

Schematic sketches of the experimental setup, the square vessel filled with the fluid and arrangements of the ultrasonic measurement lines, are shown in Figs. 1(a) top and 1(b) side view. The vessel is identical to that used in our previous studies [30,31] to which the reader is referred for a detailed description of the setup. The lateral walls of the vessel are made of 30-mm-thick PVC to attain thermal insulation. The fluid layer has a square horizontal cross section of $200 \mathrm{~mm} \times 200 \mathrm{~mm}$ and a height of $40 \mathrm{~mm}$, giving an aspect ratio of five. It is sandwiched between the top and bottom copper plates. The temperatures of each copper plate, $T_{h}$ and $T_{c}$, are controlled by water flowing through channels inside the plates. The temperature values are monitored during the experiments by thermocouples embedded in the copper plates. The vertical temperature difference is determined as $\Delta T=T_{h}-T_{c}$ and ranges from $0.36^{\circ} \mathrm{C}$ to $15.93{ }^{\circ} \mathrm{C}$. The test fluid is the eutectic alloy $\mathrm{Ga}^{67} \mathrm{In}^{20.5} \mathrm{Sn}^{12.5}$ whose kinematic viscosity is $v=3.4 \times 10^{-7} \mathrm{~m}^{2} / \mathrm{s}$ and thermal diffusivity is $\kappa=1.05 \times 10^{-5} \mathrm{~m}^{2} / \mathrm{s}$, giving $\operatorname{Pr}=0.03$ (the thermo-physical properties are summarized in Plevachuk et al. [32]). The thermal diffusion time for the fluid layer $L^{2} / \kappa$ is $153 \mathrm{~s}$. Considering a value of the density of $\rho=6360 \mathrm{~kg} / \mathrm{m}^{3}$ and a thermal expansion coefficient of $\alpha=1.24 \times 10^{-5} \mathrm{~K}^{-1}$, the Ra number can be varied in the range from $7.9 \times 10^{3}$ to $3.5 \times 10^{5}$. 


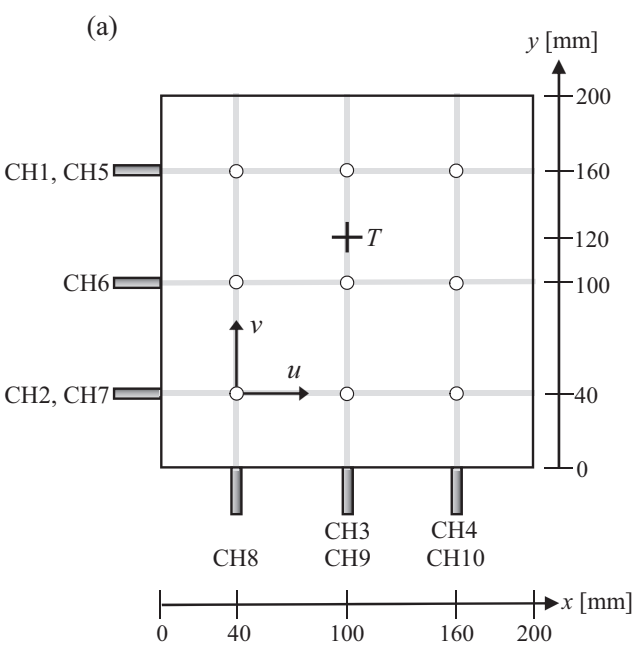

(b)

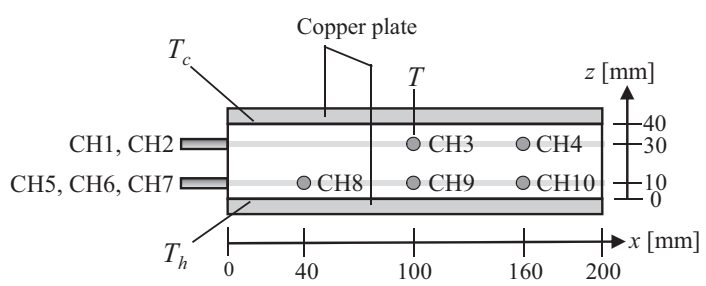

FIG. 1. Experimental setup and arrangement of measurement lines: (a) top view and (b) side view, where gray lines indicate the UVP measurement lines.

The UVP technique $[24,25]$ and thermocouples were used for obtaining instantaneous velocity profiles and pointwise temperature fluctuations of thermal turbulence. Ten ultrasonic transducers with a basic frequency of $8 \mathrm{MHz}$ were mounted horizontally inside holes drilled into the two side walls of the vessel and contacted directly with the test fluid. The gray lines shown in Fig. 1 indicate the orthogonal measuring lines. Four transducers labeled from $\mathrm{CH} 1$ to $\mathrm{CH} 4$ were installed at a distance of $10 \mathrm{~mm}$ to the top plate $(z=30 \mathrm{~mm})$, and the other six transducers labeled from CH5 to $\mathrm{CH} 10$ were set at a distance of $10 \mathrm{~mm}$ to the bottom plate $(z=10 \mathrm{~mm})$. The UVP approach provides instantaneous profiles of the velocity component projected onto each measurement line, $u(x, t)$ and $v(y, t)$, respectively. The horizontal velocity components, $(u, v)$, are obtained at the intersection points of the measurement lines represented as white circles in Fig. 1(a). The spatial resolution of the velocity measurements is $1.4 \mathrm{~mm}$ in the direction of ultrasonic beam line and $5 \mathrm{~mm}$ in the direction perpendicular to the line, whereas a temporal resolution of about $1.2 \mathrm{~s}$ has been realized. The velocity resolution is about $0.5 \mathrm{~mm} / \mathrm{s}$. The velocity profiles along each measurement line were acquired sequentially by multiplexing. A thermocouple was positioned in the fluid layer at $x=100 \mathrm{~mm}$ and $y=120 \mathrm{~mm}$, and $3 \mathrm{~mm}$ below the top plate $(z=37 \mathrm{~mm}$ ) (see $T$ in Fig. 1) to measure temperature fluctuations with a sampling rate of about $10 \mathrm{~Hz}$.

\section{MEASUREMENT RESULTS}

\section{A. Transition of coherent structures}

Systematic flow measurements were conducted in the Ra number range from $7.9 \times 10^{3}$ to $3.5 \times 10^{5}$. The analysis of the measured flow profiles discloses the existence of four different flow structures in this range. The investigation of the transitions between these coherent structures for varying Ra numbers is a key point of this study.

An oscillatory roll-like flow structure occurs at low Ra numbers around $10^{4}$. An example is shown by spatiotemporal velocity maps in Figs. $2(\mathrm{a})-2(\mathrm{~d})\left(\mathrm{Ra}=1.1 \times 10^{4}\right)$, where the velocity data were measured parallel to the $x$ axis [(a) CH1 and (c) CH6; see Fig. 1] and to the $y$ axis [(b) $\mathrm{CH} 4$ and (d) $\mathrm{CH} 9]$, respectively. The lines of $\mathrm{CH} 6$ and $\mathrm{CH} 9$ crosses at the center of the vessel at a height of $z=10 \mathrm{~mm}$, while the sensors $\mathrm{CH} 1$ and $\mathrm{CH} 4$ record the velocity at a height of $z=30 \mathrm{~mm}$. The velocity maps (a) and (c) show wavy patterns of four stripes corresponding to four rolls whose time-averaged orientations are aligned parallel to the $y$ axis. Since the profiles $\mathrm{CH} 1$ and $\mathrm{CH} 6$ were measured at different heights, they capture the top and bottom sides of the convection rolls. Thus, the 

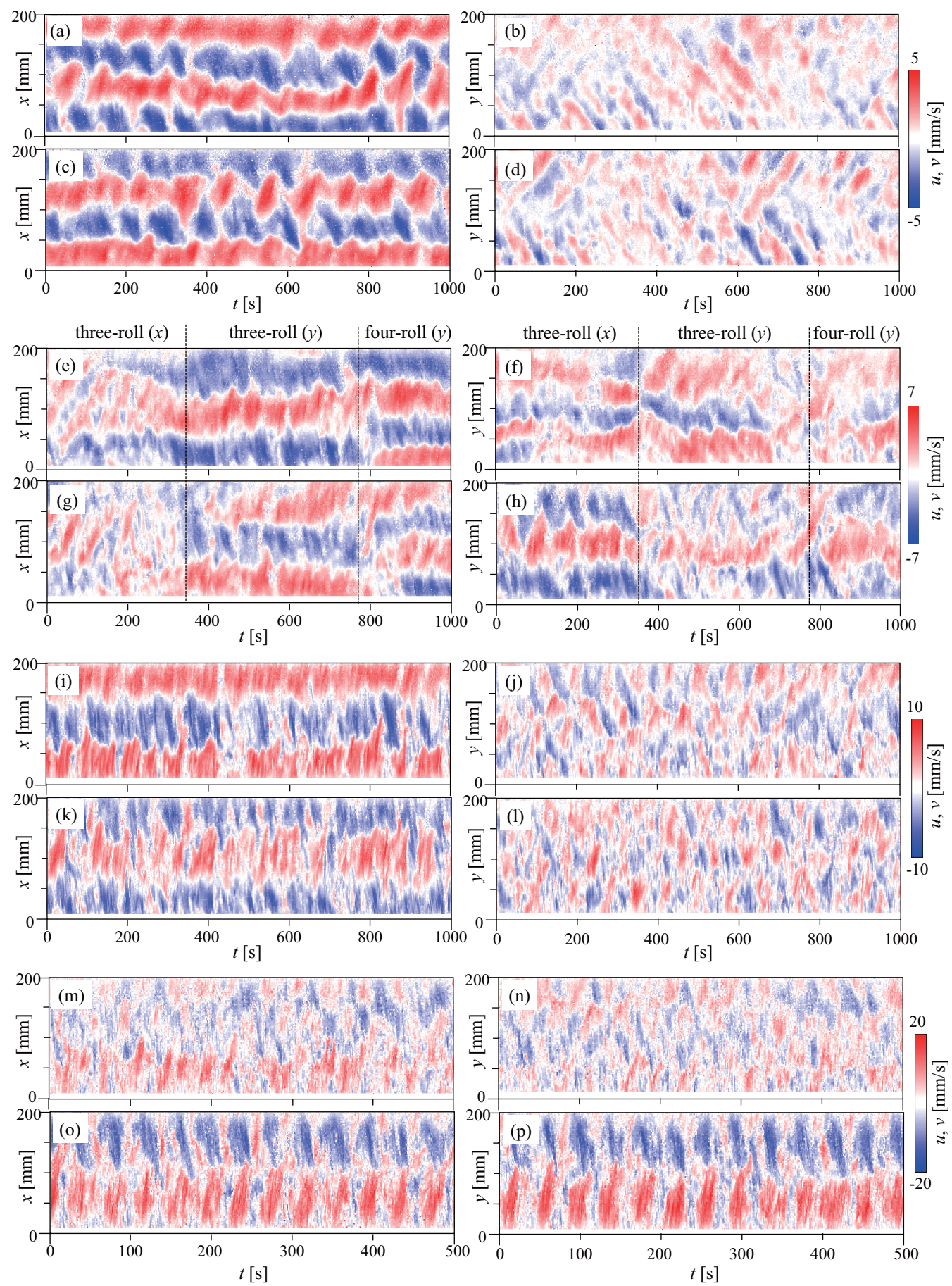

FIG. 2. Spatiotemporal velocity distributions for four cases of $\mathrm{Ra}$ along the measurement lines of transducers $\mathrm{CH} 1,4,6$, and 9 in this order in each set: (a)-(d) $\mathrm{Ra}=1.1 \times 10^{4}$ (four-roll structure), (e)-(h) $\mathrm{Ra}=2.1 \times 10^{4}$ (four-three roll transition), (i)-(l) $\mathrm{Ra}=4.6 \times 10^{4}$ (three-roll structure), and (m)-(p) $\mathrm{Ra}=$ $1.8 \times 10^{5}$ (cellular structure). 
direction of the flow velocities in the plot appears opposite. Such type of a roll pattern is observed on all other measurement lines aligned parallel to the $x$ axis. The period of the oscillation is about $100 \mathrm{~s}$. Its relation to the turnover time of the convection roll is discussed in Sec. IV B. The velocity maps (b) and (d) show the flow pattern which was measured at the measuring lines parallel to the $y$ axis. It is dominated by spots, those are smaller than $10 \mathrm{~mm}$ and synchronized with the wavy motion observed in (a) and (c). The plots suggest that these wavelike structures propagate between the wall and the interior of the vessel. The maximum velocity measured for the flow profiles along the $y$ direction is approximately half the value of that in the $x$ direction. This "four-roll structure" is stable and keeps the direction of initial roll axes throughout the measurement time over $5000 \mathrm{~s}$.

Owing to a small increase in the Ra number to $2.1 \times 10^{4}$, the roll-like structure loses its regularity with respect to the rotation axis, as shown in the spatiotemporal velocity maps in Figs. 2(e)-2(h). In this flow state, wavelike patterns are observed, which can be interpreted as four or three convection rolls whose axes are aligned alternately parallel to the $x$ or the $y$ axis. It is noticeable that the basic structure of the flow is not maintained over the entire measuring time. In the time period from $0 \mathrm{~s}$ to about $350 \mathrm{~s}$, the velocity map recorded near the side wall by transducer $\mathrm{CH} 4$ [Fig. 2(h)] shows a transformation between different structures, but at the center line (transducer CH9) shown in Fig. 2(h), a three-roll structure aligned parallel to the $x$ axis survives ["three-roll ( $x$ )" in Fig. 2; here $(x)$ means an alignment of the roll axes in the $x$ direction]. The velocity map in Fig. 2(f) shows that additional rolls emerge near the wall and then disappear after some time. The flow measurements along the $x$ axis ["three-roll (x)" in Figs. 2(e) and 2(g)] do not show a dominant roll structure at that time. The velocity field in $x$ direction looks more complicated, propagating wavelike structures are observed in the center of the fluid layer. In the time window between $350 \mathrm{~s}$ and $750 \mathrm{~s}$, three-roll structures become visible in the measurements along the $x$ direction, which means that the orientation of the convection rolls must have rotated by $90^{\circ}$ ["three-roll (y)" in Fig. 2]. This clearly shows a competition between rolls in the $x$ or $y$ direction. After around $750 \mathrm{~s}$, the velocity field shows a spontaneous transition to a wavy four-roll structure ["four-roll (y)" in Fig. 2]. All this together demonstrates that there is no stable flow in this parameter range. Over a longer period, neither three- or four-roll regimes remain stable, nor is the orientation of the rolls along the $x$ or $y$ direction constant. More complicated patterns, like rolls with an axis that runs diagonally through the fluid layer, could be formed. The transitions between the different structures occur in intervals of some hundred seconds. This state may be understood as an intermediate regime between a "four-roll structure" and a "three-roll structure"; therefore, this flow state may be referred to as a "4-3 roll transition state."

By contrast, roll-like structures with constant number of rolls are observed with further increase in $\mathrm{Ra}$ as shown in Figs. 2(i)-2(l) for $\mathrm{Ra}=4.6 \times 10^{4}$. The velocity maps (i) and (k) present a fluctuating pattern with three dominating stripes corresponding to three rolls whose axes are oriented in the $y$ direction. Compared to the regimes observed at smaller Ra numbers, the magnitude of velocity is significantly larger, and the period of the flow oscillations appears to be shorter. The maximum velocity in map (l) is about $70 \%$ of the value found in map (k). This suggests that the dominant structure is still a three-roll structure with axes in the $y$ direction, although the difference in velocity along the orthogonal measurement lines is not as great as determined in the case of smaller Ra numbers. The latter finding is a clear indication of the growing $3 \mathrm{D}$ nature of the flow. This "three-roll regime" is quasistable and keeps the direction of roll axes for some thousand seconds, but in other measurements conducted at the same Ra number one could also observe sudden changes in the direction of the rolls associated with corresponding flow reversals.

The rolllike structures completely disappear at $\mathrm{Ra}=1.8 \times 10^{5}$ as shown in Figs. $2(\mathrm{~m})-2(\mathrm{p})$. Velocity maps obtained on the center lines crossing at the central point of the vessel, presented in Figs. 2(o) and 2(p), show a strongly fluctuating flow pattern dividing the surveyed domain into two parts with respect to the center of the vessel. The fluid moves primarily from each side wall toward the center of the vessel, hence, an upwelling flow is expected in the center. According to the tendency already observed, the absolute values of the velocity continue to increase as the Ra number increases. The flow maps in Figs. 2(m) and 2(n) which were recorded along measuring lines located 
(a)

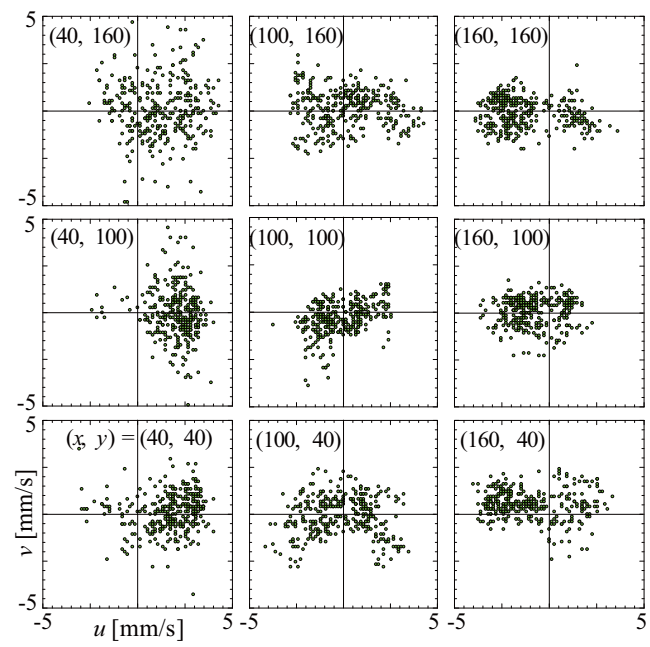

(b)

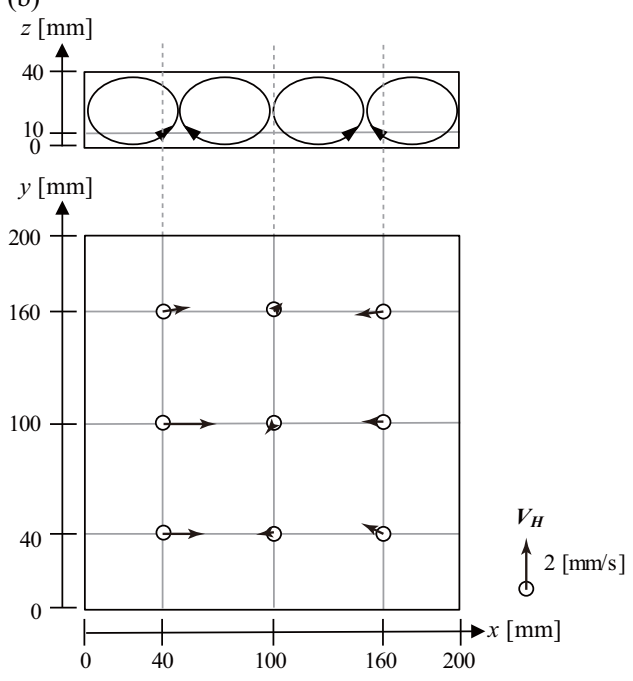

FIG. 3. (a) Scatter diagram of $(u, v)$ at nine intersection points on $z=10 \mathrm{~mm}$, and (b) time-averaged 2D velocity vectors on the horizontal plane, $V_{H}(\bar{u}, \bar{v})$, with a schematic image representing a side view of the rolllike structure expected from the results; $\mathrm{Ra}=1.1 \times 10^{4}$ and "four-roll structure" regime.

closer to the side walls show a more fragmented pattern. The magnitudes of velocities depend on the measuring position; the maximum velocities near the wall are about half of the values obtained on the center lines. The striking feature is that there is no significant difference in the flow pattern with respect to their alignment in the $x$ and $y$ directions. The main structure is, therefore, no longer composed of convection rolls, which extend over the entire length of the vessel. A new cellular structure appears.

\section{B. Details of the coherent structures}

In the present experiment, simultaneous measurements were performed using six ultrasonic transducers in a horizontal plane at $z=10 \mathrm{~mm}$ (from CH5 to CH10 in Fig. 1). As the measurement lines intersect at right angles in the fluid layer, the $2 \mathrm{D}$ velocity information $(u, v)$ can be obtained at nine intersection points. Scatter diagrams of $(u, v)$ were compiled at those intersection points, and time-averaged velocity vectors were calculated on the horizontal plane, $\boldsymbol{V}_{H}(\bar{u}, \bar{v})$, to deduce details of the 3D coherent structure for each flow regime. In Fig. 3(a) a scatter diagram for the "four-roll structure" at $\mathrm{Ra}=1.1 \times 10^{4}$ is shown [compare Figs. 2(a)-2(d)]. The number of samplings for each diagram is 320. At the intersection points, the values of the $y$ component of the velocity $v$ are nearly equally distributed around $v=0$. Corresponding distributions of the $x$ component $u$ differ significantly depending on the respective measurement line. A clear tendency for an inward flow becomes obvious on the lines at $x=40 \mathrm{~mm}$ and $x=160 \mathrm{~mm}$, while an almost equal distribution around zero can be observed at $x=100 \mathrm{~mm}$. In addition, the distributions of $u$ show a larger variance than $v$ at all points. Figure 3(b) presents a schematic view of the flow regime and the time-averaged velocity vectors, $V_{H}(\bar{u}, \bar{v})$. Assuming an equidistant four-roll regime, the center line at $x=100 \mathrm{~mm}$ runs exactly between two adjacent convection rolls, while the other two lines are located inside the outer rolls with opposite rotation. This is in accordance with the velocity vectors in Fig. 3. Obviously, the relative position of the measurement lines with respect to the convection rolls changes for the three-roll structure (see Fig. 4). These are now closer to the center of the convection rolls. Thus, the vectors in Fig. 4(b) very clearly reflect the directions of rotation of the three rolls. Moreover, it is worth to note that the scattering ranges of both $u$ and $v$ in Fig. 4(a) become wider in comparison with the four-roll structure [Fig. 3(a)], and $\boldsymbol{V}_{H}(\bar{u}, \bar{v})$ reaches larger values [Fig. 4(b)]. 
(a)

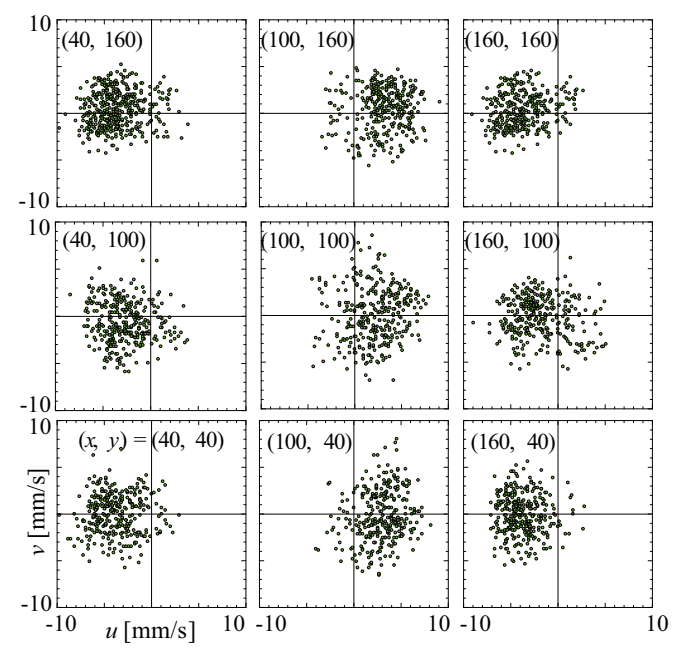

(b)

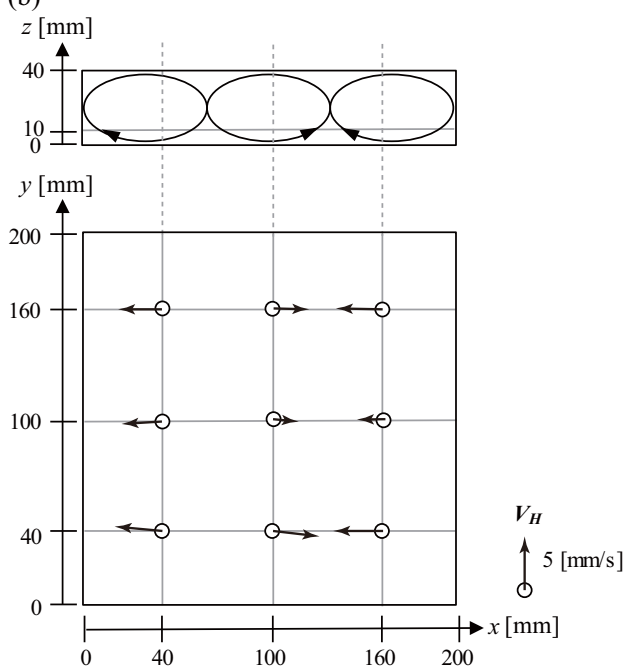

FIG. 4. (a) Scatter diagram of $(u, v)$ at nine intersection points on $z=10 \mathrm{~mm}$, and (b) time-averaged 2D velocity vectors on the horizontal plane, $\boldsymbol{V}_{H}(\bar{u}, \bar{v})$, with a schematic image representing a side view of the rolllike structure expected from the results; $\mathrm{Ra}=4.6 \times 10^{4}$ and "three-roll structure" regime.

The scatter diagrams of $(u, v)$ displayed in Fig. 5(a) correspond to the velocity maps in Figs. $2(\mathrm{~m})-2(\mathrm{p})$, which represent the cellular flow structure at $\mathrm{Ra}=1.8 \times 10^{5}$. The vectors in Fig. 5(b) reveal the characteristic pattern. Obviously, the fluid above the heating plate flows inward toward the center of the fluid layer where it rises upwards indicated by the time-averaged zero values of both velocity components at the central position. The circulation loop is closed by a diverging flow beneath the cooling plate and a descending motion towards the bottom near the midpoints of all side walls. Smaller convection cells with opposite flow direction exist apparently in the corners of the fluid vessel. The flow vectors are directed towards the corners, while the velocity magnitudes are smaller than those at the outer intersection points on the center lines. These results allow reconstructing a cellular structure in the vessel, which has upwelling flows at the center and in the four corners as the schematic view shows in Fig. 5(c). Either cellular structures with upwelling or downwelling at the center are observed for sufficiently large Ra numbers $\left(\mathrm{Ra} \geqslant 6.8 \times 10^{4}\right)$. Once one of the two cellular structures is formed, it is stable and sustained for a very long time over a few thousand seconds.

The ratio of the time-averaged root-mean-square (rms) velocities, $U_{x} / U_{y}$ recorded along the two measurement lines crossing at the center of the vessel at $z=10 \mathrm{~mm}(\mathrm{CH} 6$ and $\mathrm{CH} 9)$ are presented as a function of the Ra number in Fig. 6. The velocities are calculated according to the following formulas:

$$
U_{x}=\sqrt{\frac{1}{5 L} \int_{0}^{5 L} u^{2}(x, t) d x}, \quad U_{y}=\sqrt{\frac{1}{\frac{1}{5 L} \int_{0}^{5 L} v^{2}(y, t) d y}}
$$

Cross-bars in the equation denote the time averaging. The occurrence of the diverse coherent flow structures is marked by different symbols in the diagram. This ratio represents the ratio of horizontal velocities in the $x$ and $y$ directions. It is expected to approach very small values for perfectly 2D roll structures, while it should become close to unity for fully $3 \mathrm{D}$ coherent structures. The values of about 0.4 to 0.7 found at lower Ra numbers for the "four-roll structure" and the "three-roll structure" indicate a distinct anisotropy. The roll structures represent a tendency to two-dimensionality, although the convection rolls are subject to significant 3D disturbances. The calculation of the velocity ratio for the "four-three transition" regime was conducted in a time 
(a)
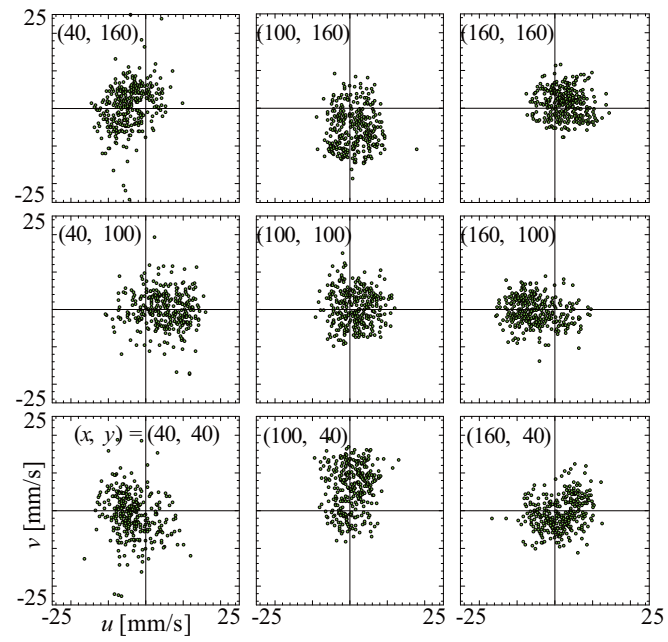

(b)

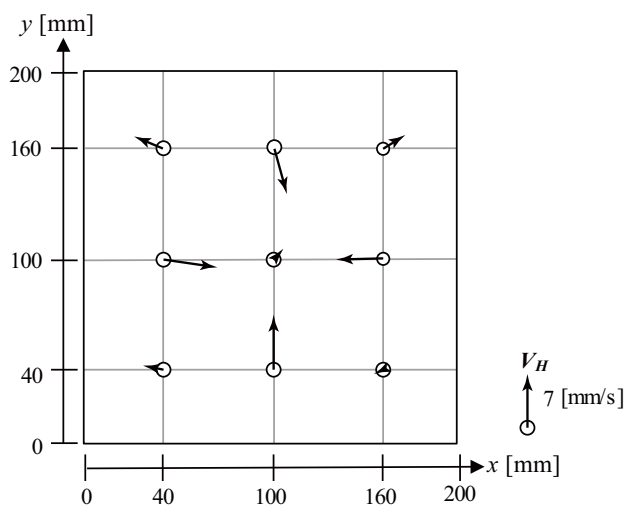

(c)



FIG. 5. (a) Scatter diagram of $(u, v)$ at nine intersection points on $z=10 \mathrm{~mm}$, (b) Time-averaged 2D velocity vectors on the horizontal plane, $\boldsymbol{V}_{H}(\bar{u}, \bar{v})$, and (c) a schematic image of 3D structure expected from the results; $\mathrm{Ra}=1.8 \times 10^{5}$ and "cellular structure" regime.

interval, at which the roll structures appear to be stable excluding flow pattern transitions. The velocity ratio reaches unity when the transition to the cellular structure occurs.

\section{Temporal characteristics of coherent structures}

In the range of Ra numbers examined in this study, quasiperiodic oscillations of the coherent structures are observed in the spatiotemporal velocity maps as mentioned in Sec. III A. The typical frequencies of dominating oscillations in the flow patterns, both rolls and cells, are obtained from the spatially averaged power spectral densities (PSDs) of the velocity maps as described below. Smoothing of the spectra obtained by a Fourier transform is achieved by dividing the velocity time series into multiple time intervals of around $200 \mathrm{~s}$. The spectra are calculated for each time interval and for each measurement point along the measurement line $\mathrm{CH} 6$, which passes through the center of the vessel. The line CH9 is considered additionally in case of the cellular structure. Then these interim results are averaged both in space and in time. The PSD for the four-roll regime at $\mathrm{Ra}=$ $1.1 \times 10^{4}$ is shown in Fig. 7(a). It has a dominant peak at $f=0.010 \mathrm{~Hz}$ corresponding to the oscillation frequency, $f_{\text {OS }}$, of the wavy flow patterns shown in the velocity maps of Figs. 2(a)-2(d). The PSD obtained for the transition regime at $\mathrm{Ra}=2.1 \times 10^{4}$ [see Fig. 7(b)] does not reveal distinct peaks as they occur in other flow regimes. The reason for this is likely the frequent change in the roll structure. The permanent three-roll regime at $\mathrm{Ra}=4.6 \times 10^{4}$ [Fig. 7(c)] again shows a clear peak at $f=0.016 \mathrm{~Hz}$. In case of the cellular structure [Ra $=1.8 \times 10^{5}$; see Fig. 7(d)], both PSDs 


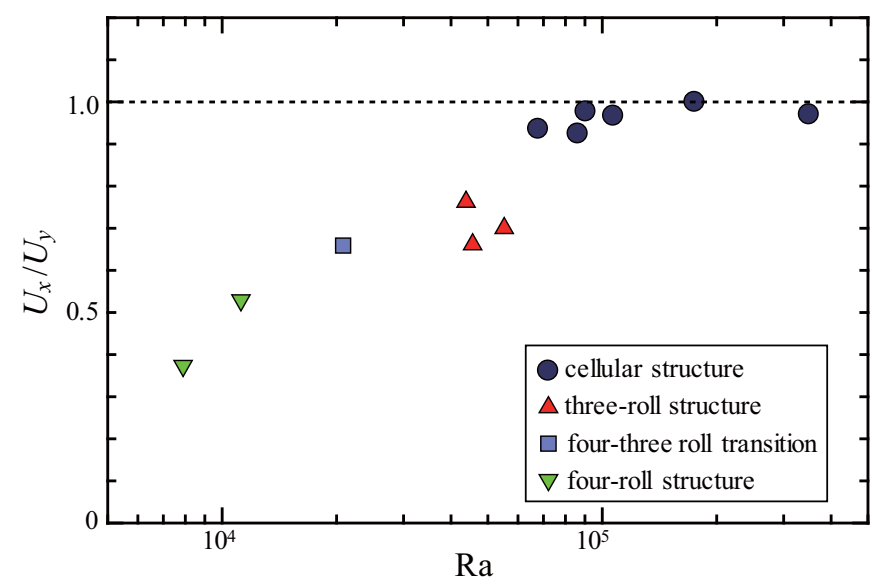

FIG. 6. Ratios of spatial rms velocities measured by $\mathrm{CH} 6$ and $\mathrm{CH} 9$ at different Ra numbers.

calculated for the orthogonal measuring lines show dominant peaks at $0.025 \mathrm{~Hz}$. In summary, it can be stated that the typical frequencies of the oscillation increase with increasing Ra, which will be discussed in further detail in Sec. IV A.

To investigate the 3D oscillations of the cellular structure in detail, time variations of $u$ at $(x, y)$ $=(50,100) \mathrm{mm}$ and $v$ at $(100,50) \mathrm{mm}$ are plotted in Fig. 8(a). The fluctuations show quasiregular oscillations corresponding to the typical frequency, $f_{\mathrm{OS}}=0.025 \mathrm{~Hz}$. Obviously, the signals of the two velocity components are in antiphase. Spatial profiles of the associated phase are calculated by Fourier transformation as displayed in Fig. 8(b). This diagram reveals a phase jump in the middle of the fluid vessel. One possible interpretation of these results is that a reciprocal elliptical deformation of the central cell as shown in Fig. 8(c) could be the reason for the oscillations observed in the measured velocity data.

\section{Statistical characteristics of temperature fluctuations}

This section focuses on the statistical characteristics of thermal turbulence derived from the temperature measurements. Temperature fluctuations measured directly in the fluid layer are shown in Figs. 9(a)-9(d). These temperature time series result from a moving average over a window
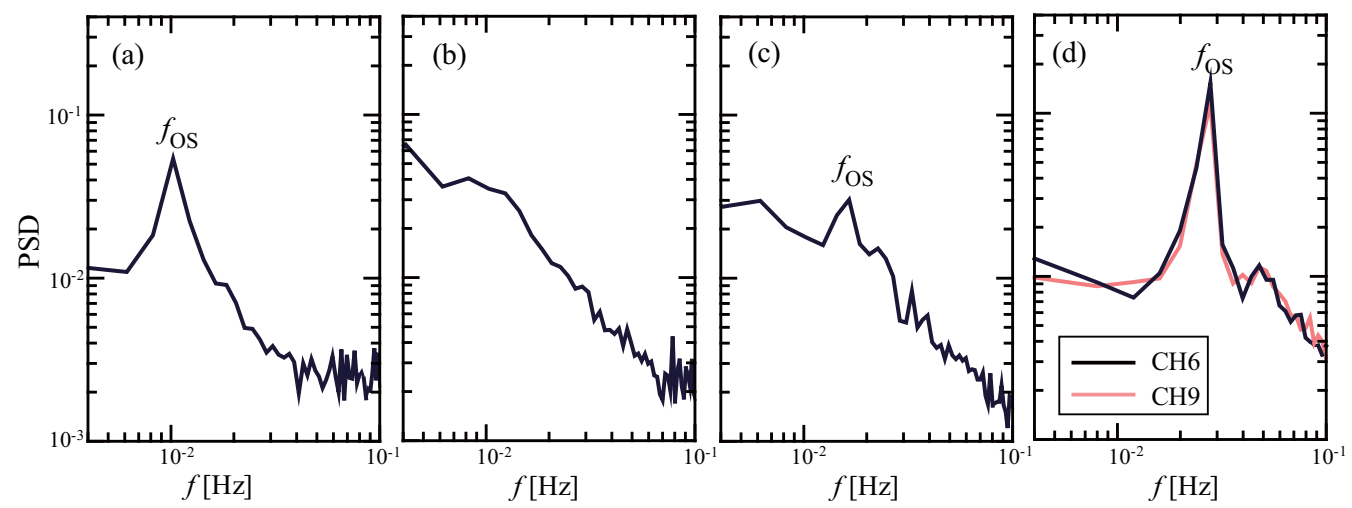

FIG. 7. Spatially averaged PSDs calculated from spatiotemporal velocity maps measured by CH6 (see Fig. 1) at (a) $\mathrm{Ra}=1.1 \times 10^{4}$ (four-roll structure), (b) $\mathrm{Ra}=2.1 \times 10^{4}$ (four-three roll transition), (c) $\mathrm{Ra}=4.6 \times 10^{4}$ (three-roll structure), and by $\mathrm{CH} 6$ and $\mathrm{CH} 9$ at (d) $\mathrm{Ra}=1.8 \times 10^{5}$ (cellular structure). 

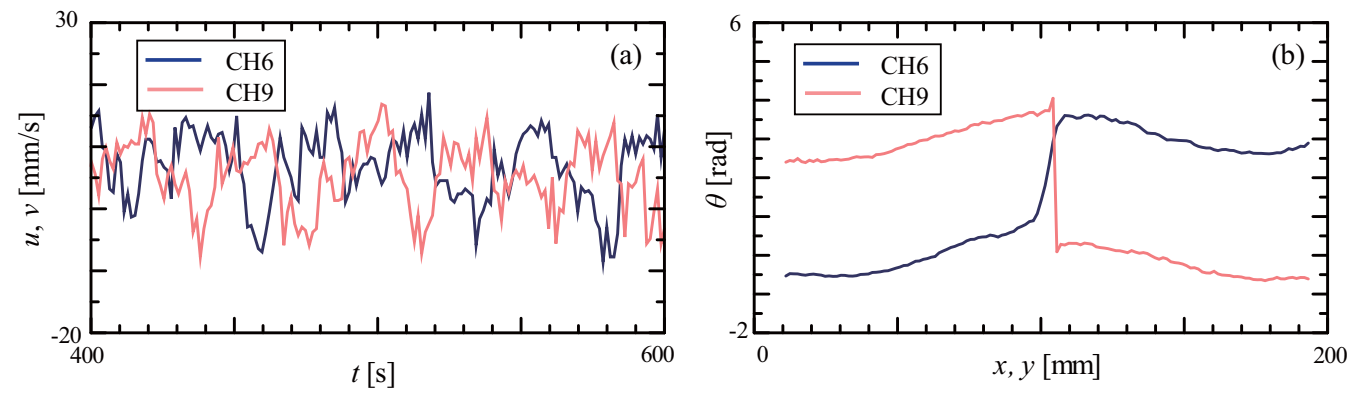

(c)
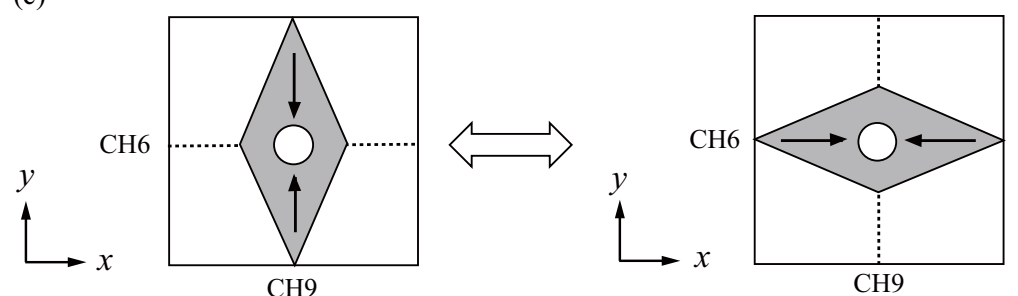

FIG. 8. (a) Time variations of $u$ at $(x, y)=(50,100) \mathrm{mm}$ measured by $\mathrm{CH} 6$ and $v$ at $(100,50) \mathrm{mm}$ measured by $\mathrm{CH} 9$ at $\mathrm{Ra}=1.8 \times 10^{5}$, (b) corresponding phase profiles at the oscillation frequency, $f_{\mathrm{OS}}=$ $0.025 \mathrm{~Hz}$, and (c) a schematic plane image of oscillations of the cellular structure.
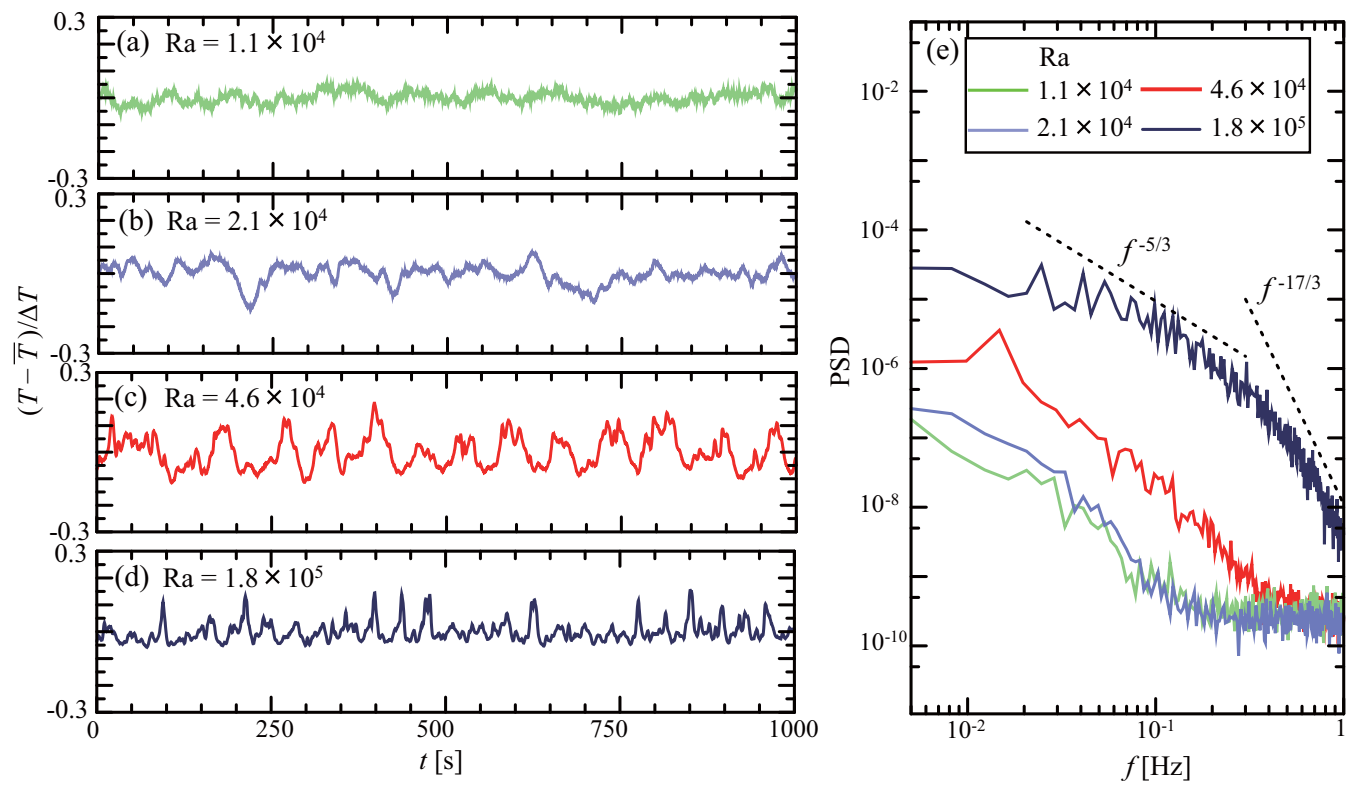

FIG. 9. Temperature fluctuations measured in the fluid layer (see Fig. 1 for the measurement point " $T$ ") at (a) $\mathrm{Ra}=1.1 \times 10^{4}$ (four-roll structure), (b) Ra $=2.1 \times 10^{4}$ (four-three transition), (c) Ra $=4.6 \times 10^{4}$ (threeroll structure), (d) $\mathrm{Ra}=1.8 \times 10^{5}$ (cellular structure), and (e) PSDs of the temperature fluctuations. The dashed lines indicate the slopes of $-5 / 3$ and $-17 / 3$. 
of $5 \mathrm{~s}$ and a normalization with the temperature difference $\Delta T$. The fluctuations obtained at $\mathrm{Ra}=1.1 \times 10^{4}$ [Fig. 9(a)] does not show such clear periodic oscillations as observed in the spatiotemporal velocity maps in Figs. 2(a) and 2(c). This is due to the fact that the temperature difference applied between the top and the bottom plate is very small for the smallest Ra number. Hence, the magnitude of the temperature fluctuations is only slightly above the noise level of the measurement. The temperature fluctuations grow with an increase in the Ra number to $2.1 \times 10^{4}$ [Fig. 9(b)]. However, no clear periodicity becomes visible, which is likely due to the distinct intermediate character of this flow regime. Pronounced quasiperiodic oscillations appear in the temperature signal at higher $\mathrm{Ra}$ numbers $\left[\mathrm{Ra}=4.6 \times 10^{4}\right.$ in Fig. 9 (c) and $\mathrm{Ra}=1.8 \times 10^{5}$ in Fig. 9(d)].

The corresponding PSDs of the temperature fluctuations are presented in Fig. 9(e). The same procedure was applied for smoothing the spectra as already described for the velocity. The thermal energy spectrum model for low-Pr turbulence predicts a $-5 / 3$ slope for the inertial-convective subrange, and $\mathrm{a}-17 / 3$ slope for the inertial-conductive subrange [33]. The corresponding slopes are indicated in the figure as dashed lines. The shape of the PSDs changes depending on Ra, and seemingly, the spectra approximate the predicted slopes with increasing Ra number. This leads to the statement that the convective motion is approaching the state of fully developed thermal turbulence for Ra numbers of $1.8 \times 10^{5}$. Herewith, it is confirmed that the cellular structure, which has been recognized as the large-scale circulation at this Ra number, occurs under the condition of thermal turbulence [3,4].

\section{DISCUSSION}

\section{A. Scaling of the size of coherent structures}

In this experiment, the wavelength of coherent flow structure increases systematically, but discretely with increasing Ra number due to the restriction by the finite horizontal dimension of the vessel. The purpose of this section is to examine whether one can derive a law for the variation of the size of coherent structures from the experimental data available in terms of flow velocity and oscillation period. In this context, the aspect ratio of the fluid vessel is expected to have a non-negligible influence. In a first step, the Ra number scaling for the characteristic velocity $U$ and the oscillation frequency $f_{\mathrm{OS}}$ is determined.

Lam et al. [34] proposed three different velocity scales as the representative velocity of the flow structure that can be estimated from limited information obtained by experiments: (i) the maximum velocity on time-averaged velocity profiles, (ii) the root-mean-square (rms) values of the velocity fluctuations, and (iii) the velocity calculated from the typical oscillation timescale of the structures. We select the rms velocity as the most robust value to be derived from experimental data. The Rayleigh number dependence of the representative flow velocity $U_{\text {rms }}$ is shown in Fig. 10(a), where the rms velocity $U_{\text {rms }}$ is calculated from spatiotemporal velocity maps as space-time rms values. The spatiotemporal velocity maps underlying the calculations are recorded along either $\mathrm{CH} 6$ or $\mathrm{CH}$, depending on which of both was aligned perpendicular to the rotation axis of rolls in the roll regimes. $U_{\mathrm{rms}}$ is converted into a nondimensional form using the thermal diffusivity $\kappa$ of the fluid and the layer thickness $L$. Different symbols in the figure indicate different regimes categorized in Sec. III A. The velocity variation can be fitted by a unified power law,

$$
U_{\mathrm{rms}} L / \kappa=0.059 \mathrm{Ra}^{0.50 \pm 0.02},
$$

where the exponent is obtained from the least square approximation of the measurement results. Similar values of the exponent around $0.5(0.46 \ldots 0.52)$ were also reported by other experimental [18,28,35] and numerical studies [36] for low-Pr number fluids. Recently, Vogt et al. [37] found a scaling law of $\mathrm{Re}=5.662 \mathrm{Ra}^{0.483}$ for convections in a gallium layer which is very close to our result in Eq. (1). From this point of view, this scaling seems quite robust, not least because the other experiments used different combinations of aspect ratios and liquid metals, in particular mercury $(\operatorname{Pr}=0.024)$ and $\Gamma=1$ [18], gallium (0.025) and $\Gamma=1.25$ [28], or gallium and $\Gamma=5$ [35]. In 

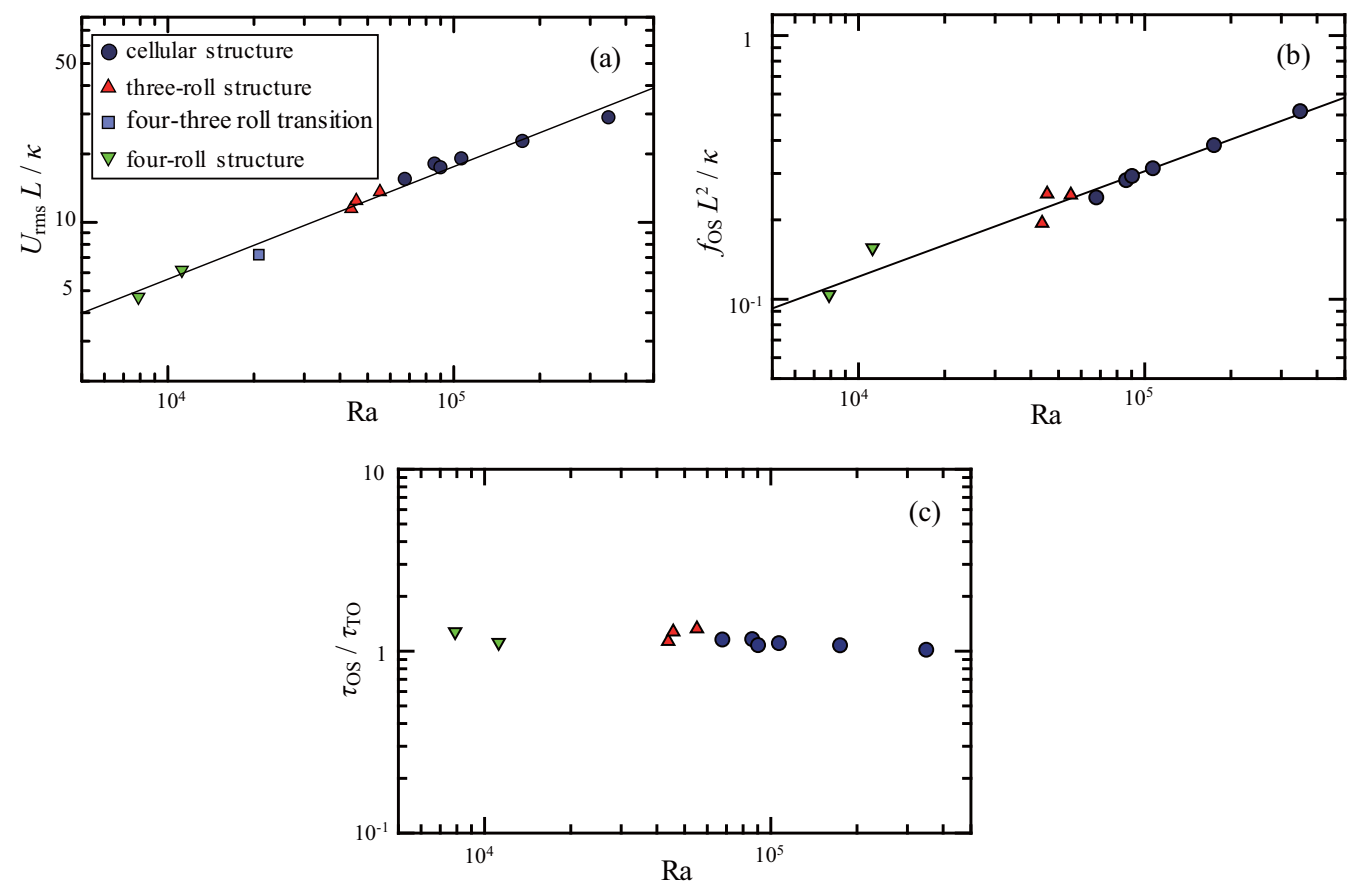

FIG. 10. Plots of (a) the representative flow velocity $U_{\text {rms }}$, where the solid line shows the fitting curve given by the least-square approximation on the plots, $U_{\mathrm{rms}} L / \kappa=0.059 \mathrm{Ra}^{0.50}$, (b) the frequency of oscillations $f_{\mathrm{OS}}$, where the solid line shows the fitting curve on the plots, $f_{\mathrm{OS}} L^{2} / \kappa=0.031 \mathrm{Ra}^{0.40}$, and (c) the periods of oscillation normalized by the turnover times $\tau_{\mathrm{TO}}$ with respect to Ra.

the last two studies the maximum velocity measured within the convection rolls was used as the representative velocity in both cases.

The typical oscillation frequency $f_{\mathrm{OS}}$ increases with the Rayleigh number as shown in Fig. 10(b), where the frequency values are obtained by the peaks on the PSDs of the velocity as described in Sec. III C. The values are normalized with the thermal diffusion timescale. The analysis gives a power law approximated as

$$
f_{\mathrm{OS}} L^{2} / \kappa=0.031 \mathrm{Ra}^{0.40 \pm 0.02}
$$

The exponent of 0.40 is slightly below the scaling laws of (i) $\mathrm{Ra}^{0.42 \pm 0.02}$ obtained from DNS data by Scheel and Schumacher [36] for $\operatorname{Pr}=0.021$ in a cylindrical cell with an aspect ratio of one and (ii) $\mathrm{Ra}^{0.419}$ given by Vogt et al. [37] for $\operatorname{Pr}=0.027$ and $\Gamma=2$.

Previous studies suggested that the period of wavy motions on convection rolls observed near the onset of convection for $\operatorname{Pr} \sim 1$ corresponds to the turnover time, the timescale of one circulation of a fluid element within a convection roll [15]. The turnover time $\tau_{\mathrm{TO}}$ is estimated in this system using the variable horizontal wavelength of the flow structure $\lambda_{\mathrm{H}}$, the fluid layer thickness $L$, and the representative flow velocity $U_{\mathrm{rms}}$ as suggested by Pandey et al. [10]:

$$
\tau_{\mathrm{TO}}=\frac{\pi\left(\lambda_{\mathrm{H}} / 4+L / 2\right)}{U_{\mathrm{rms}}},
$$

where $\pi\left(\lambda_{\mathrm{H}} / 4+L / 2\right)$ represents an estimation for the path length of a circulation assuming the perimeter of an ellipse with half of the wavelength $\lambda_{\mathrm{H}} / 2$ as the major and the layer height $L$ as the minor axis. Here the wavelength is determined from the spatial periodicity of the flow structures and turns out to be $2.5 \mathrm{~L}$ (four-roll structure), $3.3 \mathrm{~L}$ (three-roll structure) and $5 \mathrm{~L}$ (cellular structure) considering the aspect ratio $\Gamma=W / L=5$. To clarify the relation between oscillation period and 


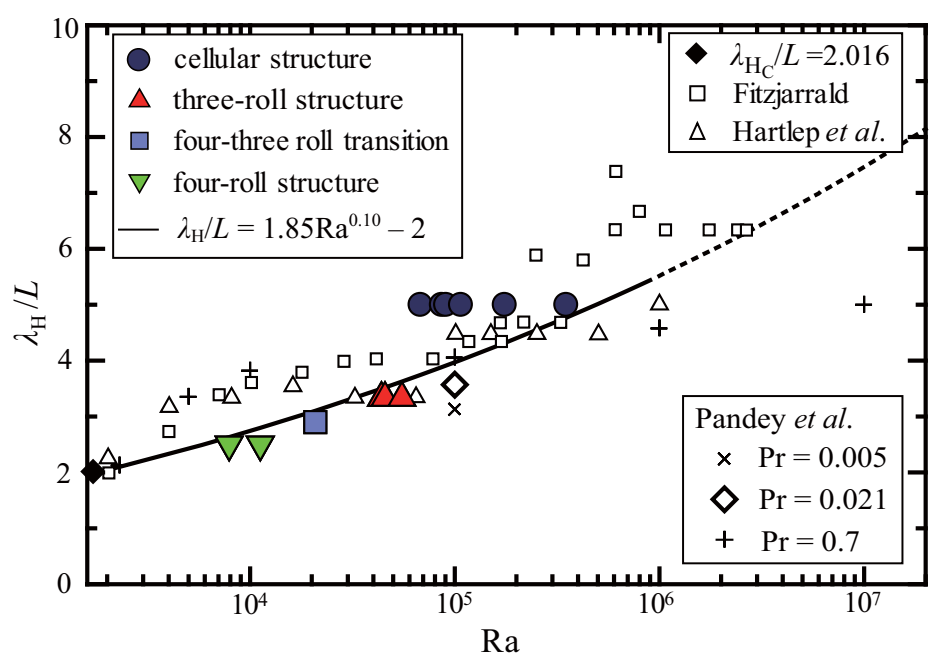

FIG. 11. Horizontal wavelength of flow structures $\lambda_{\mathrm{H}}$ versus Ra, where the solid line and the dotted line (the latter for $\mathrm{Ra}>10^{6}$ ) indicate the relation $\lambda_{\mathrm{H}} / L=1.85 \mathrm{Ra}^{0.10}-2$ [Eq. (5)]; symbols of open square, open triangle, and cross represent data points for air layers and are taken from Fitzjarrald [29], Hartlep et al. [5], and Pandey et al. [10], respectively. The diagonal cross and open diamond symbols are related to numerical results for low $\operatorname{Pr}$ numbers $(\operatorname{Pr}=0.005$ and 0.021 [10]). The symbol black diamond marks the critical wavelength $\lambda_{\mathrm{Hc}} / L=2.016$ at $\mathrm{Ra}_{\mathrm{c}}=1708$ from Chandrasekhar [40].

turnover time, the ratio $\tau_{\mathrm{OS}}=1 / f_{\mathrm{OS}}$ to $\tau_{\mathrm{TO}}$ is plotted against Ra in Fig. 10(c). It indicates

$$
\tau_{\mathrm{OS}} \approx c \tau_{\mathrm{TO}}
$$

where $c$ is constant and has a value of $\sim 1$. It is a remarkable feature that this relationship holds throughout the entire Ra number range of the present study. Obviously, this finding confirms the coherence of the large-scale flow structures in all flow regimes observed here. The coincidence of temperature and velocity oscillations and the turnover time is known as a common phenomenon in Rayleigh-Bénard convection, as it can be detected over a wide parameter range and under different aspect ratios. In particular, such behavior was observed recently for the LSC in a cylindrical vessel of aspect ratio 2 (see, for instance, Vogt et al. [37]). The oscillations at smaller aspect ratios are associated with torsional [38] or sloshing modes [39] of the LSC dynamics. It is known that a structure of parallel rolls develops after the onset of convection which shows upcoming oscillations with increasing Ra number rather soon [28]. These oscillations are related to deformations of the rolls, which give an obvious link to the turnover time. Vogt et al. [31] investigated to what extent a horizontal magnetic field determines whether these flows are 2D or 3D. This gives rise to the suggestion that the coherent structures observed in the present study are a residue of the original roll structure, although they are subject to strong disturbances. Equation (4) is a common feature for the convection in all regimes represented by Eq. (3). For deriving a scaling relation for $\lambda_{\mathrm{H}}$ as a function of the Ra number, Eqs. (1)-(3) are inserted into Eq. (4). The subsequent conversion to $\lambda_{\mathrm{H}}$ yields

$$
\lambda_{\mathrm{H}} / L \approx 2.40 / c \mathrm{Ra}^{0.10}-2
$$

The power law in Eq. (5) describes a continuous variation of $\lambda_{H}$ with Ra, although the original variation of $\lambda_{\mathrm{H}}$ obtained in the present experiment is discrete due to geometrical restrictions in the vessel of aspect ratio 5. The resulting curve of the power law for $\lambda_{\mathrm{H}}$ (taking a value of $c=1.3$ into account) is plotted in Fig. 11, including the discrete values found experimentally in the present study and the following experimental [29] and numerical data [5,10] obtained at different Pr numbers smaller than unity for comparison. A detailed discussion of this figure can be found in the next section. 


\section{B. Transitions between the coherent structures}

Four different flow structures were identified in the parameter range considered in this study. The regime diagram by Krishnamurti [1] does not suggest the existence of an oscillatory roll-like regime at $\mathrm{Ra} \sim 10^{4}$ as found in our experiments. Basically similar flow patterns consisting of convection rolls, which are subject to distinct 3D disturbances, were also observed in experiments with imposed horizontal magnetic fields carried out at similar Ra numbers in the same vessel [31]. The statistical analysis of the temperature fluctuation reveals, however, a significantly higher level of turbulence in the present case [see Fig. 9(e)], while the influence of the magnetic field obviously has a stabilizing effect on the roll structure [31]. Regardless of the high-turbulence level, the roll-like structures exist up to a Ra number of about $6 \times 10^{4}$ before a transition to a cellular structure occurs.

Hébert et al. [41] investigated the onset of Rayleigh-Bénard convection in cylindrical vessels. For aspect ratios $1.58 \leqslant \Gamma \leqslant 3.26$ just above $\mathrm{Ra}_{\mathrm{c}}$, they reported the formation of a concentric roll corresponding to an azimuthal Fourier mode with the wave number $m=0$. As already shown in Sec. III B, the flow pattern of the cellular structure resembles such a central toroidal vortex or a concentric roll, which, due to the geometry considered here, is surrounded by smaller counterrotating vortices in the corner regions of the square vessel. Strong regular oscillations are a striking feature of this regime (see Sec. IIIC). These oscillations are reflections of a mutual increase and decrease of the velocities measured in the $x$ and $y$ directions on the center lines. A simplified picture in Fig. 8 suggests a periodic elliptical deformation of the concentric roll along the two center lines of the square cross-sectional area. On the basis of the available measurement results, it is ultimately not possible to decide whether such a deformation of an originally axially symmetric structure exists or whether it is even the competition of two interacting double vortices in the $x$ and $y$ directions. In this case the strong oscillations result from the fact that each one of the double rolls dominates alternately during a half-period and the other is strongly damped at the same time. Vasiliev et al. [42] considered the dynamics of the LSC inside a cube. They describe the LSC as a superposition of two-large-scale 2D orthogonal rolls while observable phenomena such as the reorientation of LSC are due to the competition of these two rolls.

Figure 11 represents the variation of the structure size of the characteristic flow patterns with increasing Ra number. Our experimental data are compared with numerical simulations performed for square fluid layers representing different Pr numbers and aspect ratios of 25 [10] and 10 [5], respectively. Moreover, Fig. 11 contains experimental data from Fitzjarrald [29] measured in air $(\operatorname{Pr}=0.7)$ and aspect ratios between about 14 and 58. At first glance, the scaling law [Eq. (5)] gives a fairly good representation of the wave length data. The critical wavelength, $\lambda_{\mathrm{Hc}} / L=2.016$, at the critical Rayleigh number, $\mathrm{Ra}_{\mathrm{c}}=1708$ [40], is also plotted here. It becomes apparent that this point corresponds well to the suggested power law. The single numerical point for $\operatorname{Pr}=0.021$ and $\mathrm{Ra}=$ $1.0 \times 10^{5}[10]$ matches very well the experimental data. Although characterized by distinct scatter the experimental data by Fitzjarrald [29] for $\operatorname{Pr}=0.7$ seem to agree fairly good with respect to the scaling exponent but are slightly shifted towards larger values of the wavelength, which indicates that lowering the Pr number leads to smaller flow structures. This is basically confirmed by the data points provided by Pandey et al. [10] for different Pr numbers at $\mathrm{Ra}=1.0 \times 10^{5}$. On the other hand, if one considers exclusively the numerical simulations by Pandey et al. [10] and Hartlep et al. [5] for $\operatorname{Pr}=0.7$, then the situation is quite different. In the region $4 \times 10^{4} \leqslant \mathrm{Ra} \leqslant 10^{7}$, the dimensions of the structures increase only insignificantly, resulting in a much smaller exponent. This is a significant deviation from both the experimental data of Fitzjarrald [29] and the one observed here for smaller Pr numbers.

The incremental increase of the experimental data points correspond to the transitions between the flow regimes and result from the geometrical restrictions given by the fluid vessel of aspect ratio 5. In particular, the wavelength data for the cellular structure show a deviation towards smaller $\mathrm{Ra}$ numbers relative to the scaling curve. The reason for this might be the confinement by the vessel. In this regard, the numerical simulations reported by Pandey et al. [10] provide a clear indication. While the numerical results predict roll structures at $\mathrm{Ra}=1.0 \times 10^{5}$, the corresponding 
flow measurements identified the cellular structure in the experiments at $\Gamma=5$. Again, this behavior could be specific to the particular aspect ratio considered here. Besides the geometric constraints it is primarily due to our approach to determine the wave length from the spatial periodicity of the flow structures that the cellular structure does not show any changes in the wavelength with varying Ra number.

Finally, it is quite an interesting question to ask regarding the further development of the coherent structures at even higher Ra. The asymptotic state of enlargement of multiple cellular structuresalso called turbulent superstructures-was recently reported for vessels with much larger aspect ratios than that in the present study $[6,7,10,43]$. These studies suggest that a scaling as given in Eq. (5) has an upper applicable limit in terms of the Ra number also in cases of larger aspect ratios. In this respect, the experiments in this study are subject to serious limitations on the range of achievable Ra numbers and dimensions of the fluid layer. For obtaining larger structures at higher Ra numbers, the use of fluid vessels with a much larger aspect ratio is undoubtedly necessary. Based on the results obtained in this study, it can be assumed that the cellular structure found in this study might serve as a kind of unit cell for the buildup of turbulent superstructures composed of multiple cells.

\section{CONCLUSION}

The present study investigates the Rayleigh-Bénard convection inside a liquid metal layer $(\operatorname{Pr}=0.03)$ confined by a flat rectangular vessel having a square horizontal cross section and an aspect ratio of 5 . The flow structures in the vessel were characterized using the ultrasonic velocity profiling (UVP). In a range of moderate Ra numbers, $O\left(10^{4}\right)-O\left(10^{5}\right)$, the flow measurements revealed the existence of four different flow regimes. Transitions between these flow regimes occur with increasing $\mathrm{Ra}$ number accompanied by a stepwise increase of the horizontal wavelength. Accompanying measurements of the temperature fluctuations indicate that the stage of developed thermal turbulence is reached at Ra numbers $\geqslant 6.5 \times 10^{4}$. To the best of our knowledge, this study presents for the first time a systematic experimental characterization of the evolution of 3D flow structures with increasing Ra numbers for RBC in a liquid metal layer at a moderate aspect ratio.

Oscillatory roll-like structures are observed up to a Ra number of $6 \times 10^{4}$. In this parameter range there exist a four-roll and a three-roll regime as well as an unstable intermediate regime in between. It has not been expected to observe flow patterns which are dominated by convection rolls at such Ra numbers far from the critical value related to the boundary of the oscillatory instability in the Busse balloon. However, the roll-like structures survive in a moderately turbulent flow, although the intrinsically 2D roll structures were supposed to be disturbed to a considerable extent. Nevertheless, the roll-like structures observed in the present study have their origin in the convection rolls evolving as coherent structures at the onset of convection. The transition from a roll-like structure to a fully $3 \mathrm{D}$ cellular structure occurs around $\mathrm{Ra}=6.5 \times 10^{4}$. The cellular structure appears to be a typical representation of the coherent structures in thermal turbulence for the geometry considered here.

The scaling law for the spatial wavelength $\lambda_{\mathrm{H}}$ of the coherent structures was derived from the empirical relationships describing the dependency of the oscillation frequency and the representative flow velocity on the $\mathrm{Ra}$ number. Our results suggest the following scaling laws: $U_{\text {rms }} \sim \mathrm{Ra}^{0.50}$, $f_{\mathrm{OS}} \sim \mathrm{Ra}^{0.40}$, and $\lambda_{\mathrm{H}} \sim \mathrm{Ra}^{0.10}$. While the scaling exponents for the characteristic velocity and the oscillation frequency are in good agreement with previously published results, there is a discrepancy regarding the Ra number dependence of the wave length derived from our data and corresponding results obtained by DNS for thermal convection in air [5,10]. This comparison leads to the conclusion that the scaling law of the characteristic length scales with respect to the Ra number should differ significantly for the different Pr numbers.

Apart from the fact that the horizontal scale of the coherent structure increases stepwise with increasing $\mathrm{Ra}$ number, the assumption that the oscillation period of the structure is comparable to the turnover time applies to all flow regimes. The finding that the ratio between the oscillation period $1 / f_{\mathrm{O}}$ and the turnover time is about one in the Ra number range considered here confirms the coherence of the large-scale flow structures. 
Assumptions can be made concerning the evolution of the coherent flow structures for both higher Ra numbers and aspect ratios. In flat vessels with a significantly higher aspect ratio, multiple cellular structures could emerge, possibly resulting from a periodic sequence of cellular structures, as observed in this study. There is no doubt that the question regarding a potential relation between the coherent structures found in this study and turbulent superstructures is very interesting. Continuing experiments with liquid metal layers of larger aspect ratio are worthy of being the subject of further studies.

\section{ACKNOWLEDGMENTS}

The authors thank Yozo Hamano and Yasushi Takeda for fruitful discussions. Financial support of this research by the German Helmholtz Association within the framework of the HelmholtzAlliance LIMTECH is gratefully acknowledged.

[1] R. Krishnamurti, Some further studies on the transition to turbulent convection, J. Fluid Mech. 60, 285 (1973).

[2] R. Krishnamurti and L. N. Howard, Large-scale flow generation in turbulent convection, Proc. Natl. Acad. Sci. USA 78, 1981 (1981).

[3] G. Ahlers, S. Grossmann, and D. Lohse, Heat transfer and large scale dynamics in turbulent RayleighBénard convection, Rev. Mod. Phys. 81, 503 (2009).

[4] F. Chillà and J. Schumacher, New perspectives in turbulent Rayleigh-Bénard convection, Eur. Phys. J. E 35, 58 (2012).

[5] T. Hartlep, A. Tilgner, and F. H. Busse, Large Scale Structures in Rayleigh-Bénard Convection at High Rayleigh Numbers, Phys. Rev. Lett. 91, 064501 (2003).

[6] J. von Hardenberg, A. Parodi, G. Passoni, A. Provenzale, and E. A. Spiegel, Large-scale patterns in Rayleigh-Bénard convection, Phys. Lett. A 372, 2223 (2008).

[7] M. S. Emran and J. Schumacher, Large-scale mean patterns in turbulent convection, J. Fluid Mech. 776, 96 (2015).

[8] J. Bailon-Cuba, M. Emran, and J. Schumacher, Aspect ratio dependence of heat transfer and large-scale flow in turbulent convection, J. Fluid Mech. 655, 152 (2010).

[9] P. J. Sakievich, Y. T. Peet, and R. J. Adrian, Large-scale thermal motions of turbulent Rayleigh-Bénard convection in a wide aspect-ratio cylindrical domain, Int. J. Heat Fluid Flow 61, 183 (2016).

[10] A. Pandey, J. D. Scheel, and J. Schumacher, Turbulent superstructures in Rayleigh-Bénard convection, Nat. Commun. 9, 2118 (2018).

[11] F. H. Busse, Non-linear properties of thermal convection, Rep. Prog. Phys. 41, 1929 (1978).

[12] H. T. Rossby, A study of Bénard convection with and without rotation, J. Fluid Mech. 36, 309 (1969).

[13] F. H. Busse, The oscillatory instability of convection rolls in a low Prandtl number fluid, J. Fluid Mech. 52, 97 (1972).

[14] R. M. Clever and F. H. Busse, Transition to time-dependent convection, J. Fluid Mech. 65, 625 (1974).

[15] R. M. Clever and F. H. Busse, Nonlinear oscillatory convection, J. Fluid Mech. 176, 403 (1987).

[16] M. Lappa, Thermal Convection: Patters, Evolution and Stability (Wiley, Chichester, 2010).

[17] A. Nakano, H. Ozoe, and S. W. Churchill, Numerical computation of natural convection for a low-Prandtlnumber fluid in a shallow rectangular region heated from below, Chem. Eng. J. 71, 175 (1998).

[18] T. Takeshita, T. Segawa, J. A. Glazier, and M. Sano, Thermal Turbulence in Mercury, Phys. Rev. Lett. 76, 1465 (1996).

[19] S. Cioni, S. Ciliberto, and J. Sommeria, Strongly turbulent Rayleigh-Bénard convection in mercury: Comparison with results at moderate Prandtl number, J. Fluid Mech. 335, 111 (1997).

[20] T. Segawa, A. Naert, and M. Sano, Matched boundary layers in turbulent Rayleigh-Bénard convection of mercury, Phys. Rev. E 57, 557 (1998). 
[21] S. Horanyi, L. Krebs, and U. Müller, Turbulent Rayleigh-Bénard convection in low Prandtl-number fluids, Int. J. Heat Mass Trans. 42, 3983 (1999).

[22] U. Burr and U. Müller, Rayleigh-Bénard convection in liquid metal layers under the influence of a vertical magnetic field, Phys. Fluids 13, 3247 (2001).

[23] J. M. Aurnou and P. L. Olson, Experiments on Rayleigh-Bénard convection, magnetoconvection and rotating magnetoconvection in liquid gallium, J. Fluid Mech. 430, 283 (2001).

[24] Y. Takeda, Velocity profile measurement by ultrasound Doppler shift method, Intl. J. Heat Fluid Flow 7, 313 (1986).

[25] Y. Takeda (ed.), Ultrasonic Doppler Velocity Profiler for Fluid Flow (Springer, Tokyo, 2012), Vol. 101.

[26] T. Mashiko, Y. Tsuji, T. Mizuno, and M. Sano, Instantaneous measurement of velocity fields in developed thermal turbulence in mercury, Phys. Rev. E 69, 036306 (2004).

[27] Y. Tsuji, T. Mizuno, T. Mashiko, and M. Sano, Mean Wind in Convective Turbulence of Mercury, Phys. Rev. Lett. 94, 034501 (2005).

[28] T. Yanagisawa, Y. Yamagishi, Y. Hamano, Y. Tasaka, M. Yoshida, K. Yano, and Y. Takeda, Structure of large-scale flows and their oscillation in the thermal convection of liquid metal, Phys. Rev. E 82, 016320 (2010).

[29] D. E. Fitzjarrald, An experimental study of turbulent convection in air, J. Fluid Mech. 73, 693 (1976).

[30] Y. Tasaka, K. Igaki, T. Yanagisawa, T. Vogt, T. Zuerner, and S. Eckert, Regular flow reversals in RayleighBénard convection in a horizontal magnetic field, Phys. Rev. E 93, 043109 (2016).

[31] T. Vogt, W. Ishimi, T. Yanagisawa, Y. Tasaka, A. Sakuraba, and S. Eckert, Transition between quasi-twodimensional and three-dimensional Rayleigh-Bénard convection in a horizontal magnetic field, Phys. Rev. Fluids 3, 013503 (2018).

[32] Y. Plevachuk, V. Sklyarchuk, S. Eckert, G. Gerbeth, and R. Novakovic, Thermophysical properties of the liquid Ga-In-Sn eutectic alloy, J. Chem. Eng. Data 59, 757 (2014).

[33] G. K. Batchelor, I. D. Howells, and A. A. Townsend, Small-scale variation of convected quantities like temperature in turbulent fluid. Part 2. The case of large conductivity, J. Fluid Mech. 5, 134 (1959).

[34] S. Lam, X.-D. Shang, S.-Q. Zhou, and K.-Q. Xia, Prandtl number dependence of the viscous boundary layer and the Reynolds numbers in Rayleigh-Bénard convection, Phys. Rev. E 65, 066306 (2002).

[35] T. Yanagisawa, Y. Hamano, T. Miyagoshi, Y. Yamagishi, Y. Tasaka, and Y. Takeda, Convection patterns in a liquid metal under an imposed horizontal magnetic field, Phys. Rev. E 88, 063020 (2013).

[36] J. D. Scheel and J. Schumacher, Global and local statistics in turbulent convection at low Prandtl numbers, J. Fluid Mech. 802, 147 (2016).

[37] T. Vogt, S. Horn, A. M. Grannan, and J. M. Aurnou, Jump rope vortex in liquid metal convection, Proc. Natl. Acad. Sci. USA 115, 12674 (2018).

[38] D. Funfschilling and G. Ahlers, Plume Motion and Large-Scale Circulation in a Cylindrical RayleighBénard Cell, Phys. Rev. Lett. 92, 194502 (2004).

[39] E. Brown and G. Ahlers, The origin of oscillations of the large-scale circulation of turbulent RayleighBénard convection, J. Fluid Mech. 638, 383 (2009).

[40] S. Chandrasekhar, Hydrodynamic and Hydromagnetic Stability (Oxford University Press, Oxford, 1961).

[41] F. Hébert, R. Hufschmid, J. Scheel, and G. Ahlers, Onset of Rayleigh-Bénard convection in cylindrical containers, Phys. Rev. E 81, 046318 (2010).

[42] A. Vasiliev, P. Frick, A. Kumar, R. Stepanov, A. Sukhanovskii, and M. K. Verma, Mechanism of reorientations of turbulent large-scale convective flow in a cubic cell, arXiv:1805.06718v1.

[43] R. J. A. M. Stevens, A. Blass, X. Zhu, R. Verzico, and D. Lohse, Turbulent thermal superstructures in Rayleigh-Bénard convection, Phys. Rev. Fluids 3, 041501 (2018). 